\title{
An Irregular Flight Scheduling Model and Algorithm under the Uncertainty Theory
}

\author{
Deyi Mou and Wanlin Zhao \\ Institute of Mathematics for Applications, Civil Aviation University of China, Tianjin 300300, China \\ Correspondence should be addressed to Deyi Mou; deyimou@hotmail.com
}

Received 20 June 2013; Revised 15 August 2013; Accepted 18 August 2013

Academic Editor: Zhiwei Gao

Copyright (C) 2013 D. Mou and W. Zhao. This is an open access article distributed under the Creative Commons Attribution License, which permits unrestricted use, distribution, and reproduction in any medium, provided the original work is properly cited.

\begin{abstract}
The flight scheduling is a real-time optimization problem. Whenever the schedule is disrupted, it will not only cause inconvenience to passenger, but also bring about a large amount of operational losses to airlines. Especially in case an irregular flight happens, the event is unanticipated frequently. In order to obtain an optimal policy in airline operations, this paper presents a model in which the total delay minutes of passengers are considered as the optimization objective through reassigning fleets in response to the irregular flights and which takes into account available resources and the estimated cost of airlines. Owing to the uncertainty of the problem and insufficient data in the decision-making procedure, the traditional modeling tool (probability theory) is abandoned, the uncertainty theory is applied to address the issues, and an uncertain programming model is developed with the chance constraint. This paper also constructs a solution method to solve the model based on the classical Hungarian algorithm under uncertain conditions. Numerical example illustrates that the model and its algorithm are feasible to deal with the issue of irregular flight recovery.
\end{abstract}

\section{Introduction}

A schedule we have made is frequently complex and dynamic, and uncertainty fittingly characters its intrinsic nature. Various unanticipated events will disrupt the system and make the schedule deviate from its intended course, even make it infeasible; furthermore, they will bring about a large quantity of losses. Then, we apply a method of disruption management to cope with it, to reach our goals while minimizing all the negative impact caused by disruptions and to get back on track in a timely manner while effectively using available resources. The disruption management refers to the real time dynamic revision of an operational plan when a disruption occurs. This is especially important in situations where an operational plan has to be published in advance, and its execution is subject to severe random disruptions. When a published operational plan is revised, there will be some deviation cost associated with the transition from the original plan to the new plan. To reduce such deviation cost, it is essential to take them into account when generating the new plan. Disruption management is a real-time practice and often requires a quick solution when a disruption occurs. The original planning problem usually is regarded as a one-time effort, so it is practically acceptable if generating an optimal operational plan takes a dozen of minutes or hours, or even longer. However, when a disruption occurs, it is critical to immediately provide a resolution to the responsible personnel. Therefore, real-time optimization techniques are very important.

In the area of transportation network, the schedule is not frequently executed according to the original plan; the system is often disturbed because of uncertainties, time delays, stochastic perturbations and so on. It is difficult to deal with the situations. So, the complex dynamic systems are raised by Gao et al. [1] in many varieties, including the areas of transportation networks, energy generation, storage and distribution, ecosystems, gene regulation and health delivery, safety and security systems, and telecommunications. They also present various mathematical methods and techniques to discuss the issues.

Airlines spend a great deal of efforts developing flight schedules for each of their fleets, and the daily operations of an airline are strictly based on a predetermined flight schedule. So, we know how important the fleet assignment is. But there are many uncertain factors having effect on the flights, 
such as bad weather, aircraft failure, and airline traffic control. When the flight schedule is disrupted, a deviation from the flight schedule causes not only inconvenience to passenger, but also a large amount of operational cost because the airline has to make a new flight schedule that should satisfy all above constraints. So, the recovery of irregular flights faced by all airlines in the world is important and difficult to solve.

In USA, Delta Airline summed up 95,000 irregular flights, which affected 8,500,000 passengers and caused $\$ 500$ million of losses (not including satisfaction losses of passengers). Each irregular flight caused losses up to more than $\$ 5,200$ on average (not including losses to the passengers because of delays). In China, the domestic three major airlines executed $1,492,031$ flights, but there were 258,866 irregular flights, and the rate of irregular flight was $17.35 \%$ in 2008 . In the same year, the domestic airlines of small and medium sized had 176,785 flights, and 39,126 were irregular flights; the rate of irregular flight was up to $22.13 \%$. Hence, the irregular flights brought a large amount of losses to airlines and inconvenience to passenger in actual life.

Due to the complexity of irregular flight scheduling problem, it is impossible for airlines to optimize existing resources relying on experience. The problem of searching fast and efficient algorithm and software has not been solved [2]. Then Teodorović and Guberinić [3] proposed a branch and bound method to minimize total delay minutes of passengers. Teodorobic and Stojkovic [4] raised dynamic programming model based on principle of lexicographic optimization, in order to minimize the cancelled flights and the total delay minutes of passengers. Jarrah et al. [5] introduced two separate models to minimize delay minutes and cancellations, respectively, solved by critical path method (CPD). Thus, the models were not able to consider the trade-off between delay and cancellation. Gang [6] constructed a two-commodities network flow model without solving method. Yan and Yang [7] formulated a model to minimize the duration of time in which the flight scheduling was disrupted. Argüello et al. $[8,9]$ presented resource allocation path flow model for fleet assignment; the model had clear ideas describing the essence of fleet assignment, but it was difficult to solve it. The period network optimization model about flight operations recovery could be found in Bard et al. [10], and it transformed the aircraft routing problem to a network flow model depending on discrete-time. Bratu and Barnhart [11] introduced a model of flight recovery and algorithm, when irregular flight happened, considering simultaneously aircraft, crew, and passengers, to decide whether to cancel the flight or not; the aim was to minimize the sum of total operation cost of interconnection, cost of passengers, and canceling cost. The objective function wished to search the trade-off point between each cost. The essence of above models was to construct flight leg and cancelled flight or not and select the minimum cost of the program. But how to generate feasible flight routes and calculate the cost of each route were difficult. There was not a paper which found the exact optimal solution by solving model directly for airlines up to now.

During the period of irregular flights, we cannot optimize all situations, and the reasons resulting to irregular flights are frequently uncertainty. For the decision making of uncertain problem, Kouvelis and Yu [12] described robust discrete optimization to deal with decision making in environment of significant data uncertainty. Matsveichuk et al. [13-15] dealt with the flow-shop minimum-length scheduling problem with $n$ jobs processed on two machines when processing time is uncertain. Reference [15] presented minimal (maximal) cardinality of a $J$-solution generated by Johnson's algorithm to solve the problem above; however, for most generalizations of the two-machine flow-shop problem, the existence of polynomial algorithms is unlikely. For the duration of each irregular flight, we cannot analyze it without enough data, and it is not feasible to be dealt with by using stochastic programming. But we can invite experts to give the approximate duration of delay time and its uncertainty distribution. Then, we can apply uncertainty theory with a great premise. Here we will apply uncertainty theory to component model with uncertain programming and provide a stepwise algorithm for the model. In this paper, we search for minimizing the total delay minutes of passengers under the constraint of estimated cost. Next, this paper gives introduction of uncertainty theory and model, the method of constructing the model, the algorithm of solving model, and numerical example for the model. At last, we provide some future directions.

\section{Preliminaries}

In this section, some basic definitions are introduced, and the arithmetic operations of uncertain theory which needed throughout this paper are presented.

Definition 1 (Liu [16]). Let $\Gamma$ be a nonempty set and $\mathscr{L}$ a $\sigma$ algebra over $\Gamma$. Each element $\Lambda \in \mathscr{L}$ is called an event. The set function $\mathscr{M}$ is called an uncertain measure if it satisfies the following four axioms.

Axiom 1 (normality). $\mathscr{M}\{\Gamma\}=1$;

Axiom 2 (monotonicity). $\mathscr{M}\left\{\Lambda_{1}\right\} \leq \mathscr{M}\left\{\Lambda_{2}\right\}$ whenever $\Lambda_{1} \subset \Lambda_{2}$

Axiom 3 (self-duality). $\mathscr{M}\{\Lambda\}+\mathscr{M}\left\{\Lambda_{c}\right\}=1$ for any event $\Lambda$;

Axiom 4 (countable subadditivity). For every countable sequence of events $\left\{\Lambda_{i}\right\}$, we have

$$
\mathscr{M}\left\{\bigcup_{i=1}^{\infty} \Lambda_{i}\right\} \leq \sum_{i=1}^{\infty} \mathscr{M}\left\{\Lambda_{i}\right\} .
$$

Definition 2 (Liu [16]). Let $\Gamma$ be a nonempty set, $\mathscr{L}$ a $\sigma$ algebra over $\Gamma$, and $\mathscr{M}$ an uncertain measure. Then the triple $(\Gamma, \mathscr{L}, \mathscr{M})$ is called on uncertainty space.

Definition 3 (Liu [16]). An uncertain variable $\xi$ is a measurable function from the uncertainty space $(\Gamma, \mathscr{L}, \mathscr{M})$ to the set of real numbers; that is, for any Borel set $\mathbf{B}$ of real numbers, the set

$$
\{\boldsymbol{\xi} \in \mathbf{B}\}=\{\gamma \in \mathbf{\Gamma} \mid \xi(\gamma) \in \mathbf{B}\}
$$

is an event. 
For a sequence of uncertain variables $\xi_{1}, \xi_{2}, \ldots, \xi_{n}$ and a measurable function $f$, Liu [17] proved that

$$
\xi=f\left(\xi_{1}, \xi_{2}, \ldots, \xi_{n}\right)
$$

defined as $\xi(\gamma)=f\left(\xi_{1}(\gamma), \xi_{2}(\gamma), \ldots, \xi_{n}(\gamma)\right)$, for all $\gamma \in \Gamma$ is also an uncertain variable. In order to describe an uncertain variable, a concept of uncertainty distribution is introduced as follows.

Definition 4 (Liu [16]). The uncertainty distribution $\Phi$ of an uncertain variable $\xi$ is defined by

$$
\Phi(\mathbf{x})=\mathscr{M}\{\boldsymbol{\xi} \leq \mathbf{x}\}
$$

for any real number $\mathbf{x}$.

Peng and Li [18] proved that a function $\Phi: \Re \rightarrow[0,1]$ is an uncertainty distribution if and only if it is a monotone increasing function unless $\Phi(x) \equiv 0$ or $\Phi(x) \equiv 1$. The inverse function $\Phi^{-1}$ is called the inverse uncertainty distribution of $\xi$. Inverse uncertainty distribution is an important tool in the operation of uncertain variables.

Theorem 5 (Liu [16]). Let $\xi_{1}, \xi_{2}, \ldots, \xi_{n}$ be independent uncertain variables with regular uncertainty distributions $\boldsymbol{\Phi}_{1}, \boldsymbol{\Phi}_{2}, \ldots, \boldsymbol{\Phi}_{n}$, respectively. If $f\left(x_{1}, x_{2}, \ldots, x_{n}\right)$ is an increasing function with respect to $x_{1}, x_{2}, \ldots, x_{m}$ and decreasing with respect to $x_{m+1}, x_{m+2}, \ldots, x_{n}$, then

$$
\xi=f\left(\xi_{1}, \xi_{2}, \ldots, \xi_{n}\right)
$$

is an uncertain variable with inverse uncertainty distribution

$$
\begin{array}{r}
\boldsymbol{\Psi}^{-1}(\alpha)=f\left(\boldsymbol{\Phi}_{1}^{-1}(\alpha), \boldsymbol{\Phi}_{2}^{-1}(\alpha), \ldots, \boldsymbol{\Phi}_{m}^{-1}(\alpha),\right. \\
\left.\boldsymbol{\Phi}_{m+1}^{-1}(1-\alpha), \ldots, \boldsymbol{\Phi}_{n}^{-1}(1-\alpha)\right)
\end{array}
$$

Expected value is the average of an uncertain variable in the sense of uncertain measure. It is an important index to rank uncertain variables.

Definition 6 (Liu [16]). Let $\xi$ be an uncertain variable. Then the expected value of $\xi$ is defined by

$$
E[\boldsymbol{\xi}]=\int_{0}^{\infty} \mathscr{M}\{\boldsymbol{\xi} \geq r\} d r-\int_{-\infty}^{0} \mathscr{M}\{\boldsymbol{\xi} \leq r\} d r
$$

provided that at least one of the two integrals is finite.

In order to calculate the expected value via inverse uncertainty distribution, Liu [17] proved that

$$
\begin{aligned}
E[\boldsymbol{\xi}]=\int_{0}^{1} f\left(\boldsymbol{\Phi}_{1}^{-1}(\alpha), \ldots, \boldsymbol{\Phi}_{m}^{-1}(\alpha),\right. & \\
& \left.\boldsymbol{\Phi}_{m+1}^{-1}(1-\alpha), \ldots, \boldsymbol{\Phi}_{n}^{-1}(1-\alpha)\right) d \alpha
\end{aligned}
$$

under the condition described in Theorem 5. Generally, the expected value operator $E$ has no linearity property for arbitrary uncertain variables. But, for independent uncertain variables $\boldsymbol{\xi}$ and $\boldsymbol{\eta}$ with finite expected values, we have

$$
E[a \boldsymbol{\xi}+b \boldsymbol{\eta}]=a E[\boldsymbol{\xi}]+b E[\boldsymbol{\eta}]
$$

for any real numbers $a$ and $b$.

Theorem 7 (Liu [16]). Assume the objective function $f\left(\mathbf{x}, \xi_{1}\right.$, $\left.\xi_{2}, \ldots, \xi_{n}\right)$ is strictly increasing with respect to $\xi_{1}, \xi_{2}, \ldots, \xi_{m}$ and strictly decreasing with respect to $\xi_{m+1}, \xi_{m+2}, \ldots, \xi_{n}$. If $\xi_{1}$, $\xi_{2}, \ldots, \xi_{n}$ are independent uncertain variables with uncertainty distribution $\Phi_{1}, \Phi_{2}, \ldots, \Phi_{n}$, respectively, then the expected objective function

$$
E\left[f\left(\mathbf{x}, \xi_{1}, \xi_{2}, \ldots, \xi_{n}\right)\right]
$$

is equal to

$$
\begin{aligned}
\int_{0}^{1} f\left(\mathbf{x}, \boldsymbol{\Phi}_{1}^{-1}(\alpha), \ldots, \boldsymbol{\Phi}_{m}^{-1}(\alpha)\right. & \\
& \left.\boldsymbol{\Phi}_{m+1}^{-1}(1-\alpha), \ldots, \boldsymbol{\Phi}_{n}^{-1}(1-\alpha)\right) d \alpha .
\end{aligned}
$$

Theorem 8 (Liu [16]). Assume $f\left(\mathbf{x}, \xi_{1}, \xi_{2}, \ldots, \xi_{n}\right)$ is strictly increasing with respect to $\xi_{1}, \xi_{2}, \ldots, \xi_{m}$ and strictly decreasing with respect to $\xi_{m+1}, \xi_{m+2}, \ldots, \xi_{n}$, and $g_{j}\left(\mathbf{x}, \xi_{1}, \xi_{2}, \ldots, \xi_{n}\right)$ are strictly increasing with respect to $\xi_{1}, \xi_{2}, \ldots, \xi_{k}$ and strictly decreasing with respect to $\xi_{k+1}, \xi_{k+2}, \ldots, \xi_{n}$, for $j=1,2, \ldots, p$. If $\xi_{1}, \xi_{2}, \ldots, \xi_{n}$ are independent uncertain variables with uncertainty distributions $\Phi_{1}, \Phi_{2}, \ldots, \Phi_{n}$, respectively, then the uncertain programming

$$
\begin{array}{ll}
\min _{x} & E\left[f\left(\mathbf{x}, \xi_{1}, \xi_{2}, \ldots, \xi_{n}\right)\right], \\
\text { s.t. } & \mathscr{M}\left\{g_{j}\left(\mathbf{x}, \xi_{1}, \xi_{2}, \ldots, \xi_{n}\right) \leq C\right\} \geq \alpha_{j}, \quad j=1,2, \ldots, p,
\end{array}
$$

is equivalent to the crisp mathematical programming

$$
\begin{array}{ll}
\min _{x} & \int_{0}^{1} f\left(\mathbf{x}, \boldsymbol{\Phi}_{1}^{-1}(\alpha), \ldots, \boldsymbol{\Phi}_{m}^{-1}(\alpha),\right. \\
& \left.\boldsymbol{\Phi}_{m}+1^{-1}(1-\alpha), \ldots, \boldsymbol{\Phi}_{n}^{-1}(1-\alpha)\right), \\
\text { s.t. } \quad & g_{j}\left(\mathbf{x}, \boldsymbol{\Phi}_{1}^{-1}\left(\alpha_{j}\right), \ldots, \boldsymbol{\Phi}_{k}^{-1}\left(\alpha_{j}\right), \boldsymbol{\Phi}_{k+1}^{-1}\left(1-\alpha_{j}\right), \ldots,\right. \\
& \left.\boldsymbol{\Phi}_{n}^{-1}\left(1-\alpha_{j}\right)\right) \leq C, \quad j=1,2, \ldots, p .
\end{array}
$$

\section{Problem Description}

For airlines, irregular flights are not expected but they are inevitable frequently owing to objective factors in practice. So, each airline has its own methods to deal with the issue. At present, the irregular flight recovery is a complex and huge project to airlines. Generally speaking, the Airline 


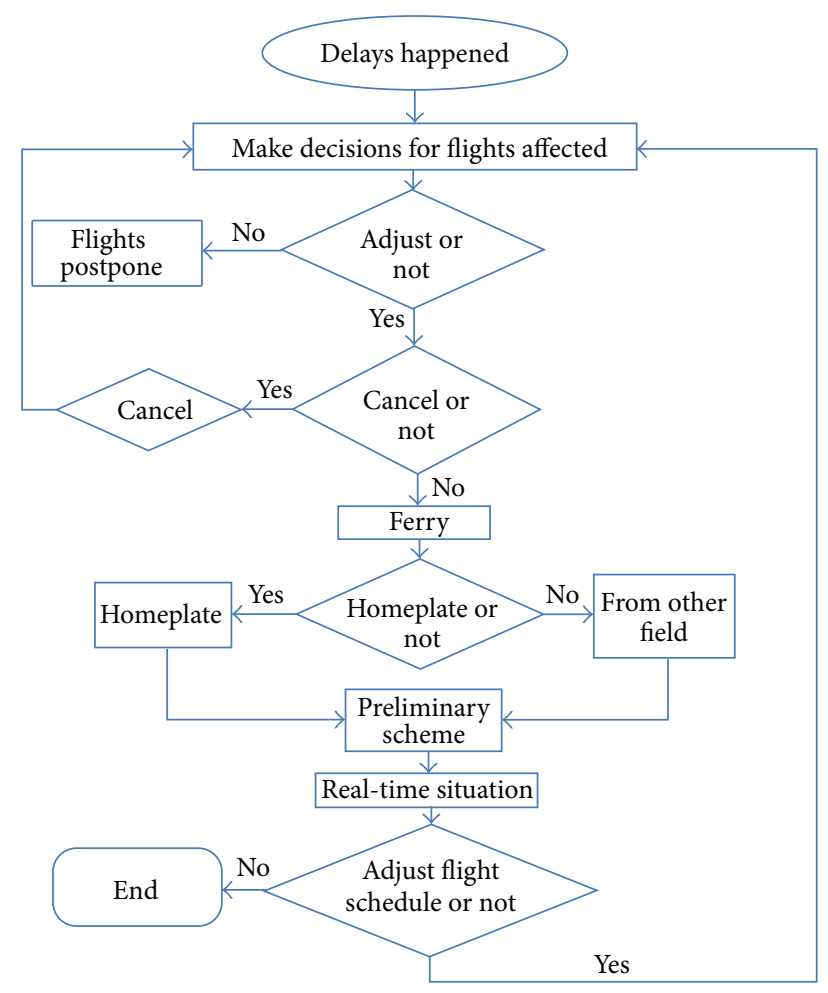

FIgURE 1: Flow chart of procedure.

Operational Control (AOC) of each airline takes the following procedure for dealing with the irregular flights.

Step 1. Maintenance Control Center (MCC) reports delays to AOC.

Step 2. AOC gets detailed information from relevant departments.

Step 3. AOC gives the expected minutes of flight delay according to the information.

Step 4. AOC makes a decision whether the first delay flight cancels or postpones.

Step 5. Making decisions to the following flights.

Step 6. AOC always pays attention to real-time situations and whether the decision will be to remake or not based on realtime changes.

The flow chart of procedure is shown in Figure 1. From this flow chart, we can get that it is difficult to make a suitable and reasonable schedule. In this paper, we resolve the irregular flight problem about fleet reassignment based on the flow chart. When irregular flights happen, the durations of delay time only are predicted through the data given by experts, in order to help the decision makers to reassign fleets better. During the period of delay time, the airlines can postpone the flights, minimize the delay minutes or delay cost by reassigning and canceling flights, or minimize the total delay minutes under the constraint of estimated cost by reassigning flights; in a word, the aim is to serve the passengers better.
Tobias [19] presented a tabu search and a simulated annealing approach to the flight perturbation problem and used a tree-search algorithm to find new schedules for airlines, and it could be successfully used to solve the flight perturbation problem. Gao et al. [20] put forward a greedy simulated annealing algorithm which integrated the characteristics of GRASP and simulated annealing algorithm, and the algorithm was able to solve large-scale irregular flight schedule recovery. Xiuli [21] introduced a stepwise-delay algorithm to research irregular flight problem. She solved the problem from the delay cost and delay minutes, respectively. In the paper, the delay minutes and delay cost were set down as constant values. From comparing the two methods, Xiuli got the result that to construct the model with delay minutes obtained successful results.

But there are not enough data of irregular flights to analyze; the data is difficult to deal with under the stochastic condition. In actual situations, we cannot obtain enough data to analyze, so, in this paper, we treat the duration of delay time of aircraft as an uncertain variable during the period of irregular flights happening, and its distribution is given by experts. Under the constraints of estimated cost and aircraft assignment model, the objective function is to minimize the total delay minutes of passengers relying on irregular flights. Then we solve the model and get the solution.

\section{Model Development}

In actual situations, the factors which cause irregular flights are uncontrolled frequently. Especially when the weather is bad and aircraft failure happens, the durations of bad weather and aircraft failure cannot be predicted exactly because of lacking enough data under stochastic condition. In this model, we consider the delay time as an uncertain variable, and its uncertainty distribution is given by experts.

Firstly, we introduce the following notations to present the mathematical formulation throughout the remainder of this paper.

\section{Indices, Sets, and Parameters}

$f$ : index for set of flights $F$

$s$ : index for set of airports $S$

$i$ index for set of types of aircrafts $I$

I: set of types of aircrafts

$F$ : set of flights

$S$ : set of airports

$A$ : set of available aircrafts

$t_{i}$ : ready time of aircraft $i$

$t_{f}^{d}$ : planning departure time of flight $f$

$t_{f}^{a}$ : planning arrival time of flight $f$

$t_{i}^{f}$ : uncertain delay time of flight $f$ executed by type $i$ of aircraft, $t_{i}^{f} \sim \varphi_{i}(t), i \in A$

$p_{f}^{b}, p_{f}^{e}$ : reservation number of business and economy class in flight $f$ 
$q_{f}^{b}, q_{f}^{e}$ : fare of a ticket of business and economy class in flight $f$

$n_{f}$ : total reservations of flight $f$

$u$ : disappointment rate of passengers

$v$ : losses value of passengers per minute

$p_{i}^{f}$ : delay loss of flight $f$ executed by aircraft $i, p_{i}^{f}=$ $w n_{f} t_{i}^{f}$, when $t_{i}^{f}>0$, or $=0$

$P_{f}$ : cost of canceling flight $f$

$\beta$ : airport usage charge per minute

$\gamma$ : cost of depreciation of aircraft per minute

$c_{i}^{f}$ : delay cost of flight $f$ executed by aircraft $i$

$c_{0}^{f}$ : estimated cost of flight $f$

$C^{f}=\left(c_{i}^{f}\right), C_{0}=\left(c_{0}^{f}\right)$

$\alpha_{0}$ : confidence level

$k$ : weight of cost of cancelling flight

$x_{i}^{f}$ : binary variable, $=1$ if flight $f$ is executed by aircraft $i$ and 0 otherwise

$y_{f}$ : binary variable, $=1$ if flight $f$ is canceled and 0 otherwise.

Xiuli [21] presented two models to deal with the irregular flight. Their objective functions were to minimize delay minutes and total delay cost:

$$
\begin{aligned}
& \min \left(\sum_{f \in F} \sum_{i \in A} x_{i}^{f} p_{i}^{f}+\sum_{f \in F} k n_{f} y_{f}\right), \\
& \min C^{f}\left(\mathbf{x}, t^{f}\right) .
\end{aligned}
$$

In our paper, we construct the model under the basis of the two models, and integrate them to one. Next, based on the analysis of the decision making process, we integrate the estimated cost and the total delay minutes of passengers model and propose the following model:

$$
\begin{array}{ll}
\min & E\left\{\sum_{f \in F} \sum_{i \in A} x_{i}^{f} p_{i}^{f}+\sum_{f \in F} k n_{f} y_{f}\right\}, \\
\text { s.t. } & \mathscr{M}\left\{C^{f}\left(\mathbf{x}, t^{f}\right)<C_{0}\right\} \geq \alpha_{0}, \\
& \sum_{i \in A} x_{i}^{f}+y_{f}=1, \quad \forall f \in F, a, d \in S, \\
& c_{i}^{f}=\left\{x_{i}^{f}\left(p_{f}^{e} \times q_{f}^{e}+p_{f}^{b} \times q_{f}^{b}\right) \times u\right. \\
& \left.+(\beta+\gamma) t_{i}^{f}+p_{f} y_{f}\right\}, \\
& \sum_{f \in F} x_{i}^{f} \leq 1, \quad \forall i \in A, \\
& x_{i}^{f} \in\{0,1\}, \quad y_{f} \in\{0,1\} .
\end{array}
$$

In the model, (15) is the objective function of minimizing the total delay minutes of passengers, the former is the delay minutes depending on the irregular flights, the latter is the equivalent delay minutes relying on flights cancelled; (16) is the constraint of estimated cost; (17) states a flight is either flown once by an aircraft or canceled; (18) is the delay cost of flight $f$ executed by aircraft $i$; (19) assigns no more than one aircraft to execute flight $f$; and (20) is the integer constraint of $0-1$.

The model is to minimize the total delay minutes of passengers under the estimated cost. Generally speaking, we need to recur to intelligent algorithm to solve the model; it is a huge project to get its solution [22].

But, it will be much easier under the uncertainty theory to deal with the problem. Note that $C^{f}\left(\mathbf{x}, t^{f}\right)$ is strictly increasing with respect to $t_{i}^{f}(i=1,2, \ldots, n)$, and $t_{1}^{f}, t_{2}^{f}, \ldots, t_{n}^{f}$ are independent uncertain variables with uncertainty distributions $t_{i}^{f} \sim \varphi_{i}(t)(i=1,2, \ldots, n)$, respectively. According to Theorem 8 , the above model will be equivalent to the following deterministic model:

$$
\begin{array}{ll}
\min \quad & \left\{\sum_{f \in F} \sum_{i \in A}\left(x_{i}^{f} w n_{f} \int_{0}^{1} \varphi_{i}^{-1}\left(t_{i}^{f}, \alpha\right) d \alpha\right)\right. \\
& \left.+\sum_{f \in F} k n_{f} y_{f}\right\}, \\
\text { s.t. } \quad \Phi^{-1}\left(\mathbf{x}, \alpha_{0}\right)<C_{0}, \\
& \sum_{i \in A} b_{f}^{d i} b_{f}^{a i} x_{i}^{f}+y_{f}=1, \quad \forall f \in F, a, d \in S, \\
& c_{i}^{f}=\left\{x_{i}^{f}\left(p_{f}^{e} \times q_{f}^{e}+p_{f}^{b} \times q_{f}^{b}\right) \times u\right. \\
& \left.+\beta t_{i}^{f}+p_{f} y_{f}\right\}, \quad y_{f}=1-x_{i}^{f}, \\
& \sum_{f \in F} x_{i}^{f} \leq 1, \quad \forall i \in A, \\
& x_{i}^{f} \in\{0,1\}, \quad y_{f} \in\{0,1\} .
\end{array}
$$

In this model, the objective function is to minimize the expected total delay minutes of passengers, and the estimated cost is treated as a chance constraint, where

$$
\boldsymbol{\Phi}^{-1}(\mathbf{x}, \alpha)=\left(\varphi_{i}^{-1}\left(t_{i}^{f}, \alpha\right)\right) .
$$

\section{Solution Method and Complexity}

5.1. Solution Method. In the model, there is an objective function, but it contains two kinds of decision variables $x_{i}^{f}$ and $y_{f}$. To solve the model, we make use of a stepwise-delay algorithm. The procedure of solution is as follows.

Step 1. Based on the information postponed, getting the timetable of flights as $Y W$. 
TABLE 1: Estimated cost.

\begin{tabular}{lc}
\hline$f$ & $c_{0} / \mathrm{RMB}$ \\
\hline $\mathrm{xx} 1337$ & 55224 \\
$\mathrm{x} x 1338$ & 52452 \\
$\mathrm{xx} 1223$ & 63540 \\
$\mathrm{xx} 1224$ & 88740 \\
$\mathrm{xx} 1209$ & 36546 \\
$\mathrm{xx} 1519$ & 26760 \\
$\mathrm{xx} 1210$ & 34880 \\
\hline
\end{tabular}

Step 2. Sorting the delay flights depending on original departure time from the timetable of delay flight, and searching the first airport where delay happened. During the delay period, we retrieve the serial number of aircrafts through the airport, and note them down in the table $\mathrm{CH}$.

Step 3. Finding available aircrafts in the delay airport, a time permutation table $T$ is built via the constraints, $\Phi^{-1}(\mathbf{x}, \alpha)<$ $C_{0}$. The delay minutes are replaced by $E\left[t_{i}^{f}\right]$; then we can get the following:

$$
T=\left(E\left[t_{i}^{f}\right]\right)=\begin{array}{cccc}
1 * & 2 * & \cdots & n * \\
f_{1} & f_{2} \\
\vdots & f_{n}
\end{array}\left(\begin{array}{cccc}
E\left[t_{1}^{1}\right] & E\left[t_{1}^{2}\right] & \cdots & E\left[t_{1}^{n}\right] \\
E\left[t_{2}^{1}\right] & E\left[t_{2}^{2}\right] & \cdots & E\left[t_{2}^{n}\right] \\
\vdots & \vdots & \vdots & \vdots \\
E\left[t_{n}^{1}\right] & E\left[t_{n}^{2}\right] & \cdots & E\left[t_{n}^{n}\right]
\end{array}\right) .
$$

Step 4. For T, we use Hungarian algorithm to reassign available aircraft and get the new timetable:

$$
p=\begin{gathered}
1 * \\
f_{1} \\
f_{2} \\
\vdots \\
f_{n}
\end{gathered} \quad\left(\begin{array}{cccc}
1 & 0 & \cdots & 0 \\
0 & 0 & \cdots & 0 \\
\vdots & \vdots & \vdots & \vdots \\
0 & 1 & \cdots & 0
\end{array}\right)
$$

Step 5. Renewing the $Y W$, the relevant aircraft assignment is replaced by the consequence from Step 4; then turn to Step 2, and steps are repeated until there are no delay flights or optimal flights. Then the results are put out.

5.2. Complexity. For one delay airport, we use Hungarian algorithm to reassign fleets in that the complexity is $O\left(n^{2}\right)$. When the number of delay airports is $m$, the algorithm will be iterated in each airport, so that the total complexity is $O\left(m n^{2}\right)$ which is a polynomial. So it is a feasible method in applications.

\section{Illustrative Example and Computational Result}

In order to test the model and the solution algorithms applied in the actual situation, we perform numerical tests based on the domestic operation department with reasonable assumptions.

We suppose that the airline has the hub airport of $s_{1}$; the flights are $s_{1}$ to $s_{2}, s_{1}$ to $s_{3}$, and $s_{2}$ to $s_{3}$. Its types of aircrafts are $A 310$ and B737. Assume that the delay minutes of irregular flights are linear uncertain variables, and their distributions are as follows:

$$
\begin{aligned}
& t_{1} \sim \mathscr{L}(10: 40,11: 20), \quad t_{2} \sim \mathscr{L}(15: 50,16: 30), \\
& t_{3} \sim \mathscr{L}(12: 10,12: 50), \quad t_{4} \sim \mathscr{L}(15: 10,15: 50) \text {, } \\
& t_{5} \sim \mathscr{L}(16: 20,17: 00) \text {. }
\end{aligned}
$$

The disappointment rate of passengers $u=0.07 t_{i}^{f} / 60+$ 0.4. The delay minutes of passengers are as follows:

$$
p_{i}^{f}= \begin{cases}0.3 n_{f} \times \frac{t_{i}^{f}}{60} & t_{i}^{f} \in[0,60), \\ 0.5 n_{f} \times \frac{t_{i}^{f}}{60} & t_{i}^{f} \in[60,120), \\ 0.7 n_{f} \times \frac{t_{i}^{f}}{60} & t_{i}^{f} \in[120,240), \\ 0.9 n_{f} \times \frac{t_{i}^{f}}{60} & t_{i}^{f} \in[240, \infty) .\end{cases}
$$

We assume that the constraint of estimated cost is shown in Table 1.

At last, we assume that the timetable is shown in Table 2.

The predetermined confidence level $\alpha_{0}=0.9$.

Then, we use the algorithm and get the optimal solution shown in Table 3.

Comparing Tables 1, 2 and 3, we can get the optimal solution through the model. From Table 3, we can see that the cost is 196,637 RMB under the constraint of 358,142 RMB, and the flight delay time is ten minutes less than Table 2. Based on Tables 3 and 2, there is a great difference in the total delay minutes of passengers. Table 2 is about 5.5 times as many as Table 3, and there is not a cancelled flight in Table 3. So the reassignment of Table 3 is much better than Table 2 . The example shows that the model and algorithm can get a method of flight recovery better.

\section{Conclusions and Future Directions}

The irregular flights always happen in actual situations; in order to deal with the issue, in this paper, we developed a model for fleet reassignment based on uncertain programming during the period of irregular flights and presented a stepwise optimization method strategy based on Hungarian 
TABLE 2: Timetable postponed.

\begin{tabular}{|c|c|c|c|c|c|c|c|c|c|}
\hline$i$ & $f$ & $t_{f}^{d}$ & $t_{f}^{a}$ & $t_{i}$ & $E\left(t_{i}^{f}\right) /$ minute & $p_{f}^{b} / q_{f}^{b}$ & $p_{f}^{e} / q_{f}^{e}$ & $b_{f}^{d i}$ & $b_{f}^{a i}$ \\
\hline $310 / 3912$ & xx3108 & $8: 00$ & $10: 30$ & $8: 00$ & 0 & $110 / 700$ & $8 / 1400$ & $s_{1}$ & $s_{2}$ \\
\hline $310 / 3912$ & xx3109 & $11: 00$ & $13: 30$ & 11:00 & 0 & $120 / 700$ & $8 / 1400$ & $s_{2}$ & $s_{1}$ \\
\hline $737 / 3010$ & xx1337 & $9: 50$ & $14: 20$ & $t_{1}$ & 70 & $144 / 1800$ & $9 / 3600$ & $s_{1}$ & $s_{3}$ \\
\hline $737 / 3010$ & xx1338 & $15: 00$ & $19: 30$ & $t_{2}$ & 70 & $135 / 1800$ & $8 / 3600$ & $s_{3}$ & $s_{1}$ \\
\hline $737 / 3023$ & xx1209 & 11:00 & $13: 30$ & $t_{3}$ & 90 & $157 / 700$ & $10 / 1400$ & $s_{1}$ & $s_{2}$ \\
\hline $737 / 3023$ & xx1210 & $14: 00$ & $16: 30$ & $t_{4}$ & 90 & $140 / 700$ & $10 / 1400$ & $s_{2}$ & $s_{1}$ \\
\hline $737 / 3982$ & xxl223 & $12: 00$ & $16: 30$ & $0: 00$ & Canceled & $175 / 1800$ & $10 / 3600$ & $s_{1}$ & $s_{3}$ \\
\hline $737 / 3982$ & xx1224 & $17: 00$ & $21: 30$ & $0: 00$ & Canceled & $275 / 1800$ & $10 / 3600$ & $s_{3}$ & $s_{1}$ \\
\hline $310 / 8010$ & xx1518 & $8: 00$ & $12: 30$ & $8: 00$ & 0 & $130 / 1800$ & $10 / 3600$ & $s_{3}$ & $s_{1}$ \\
\hline $310 / 8010$ & xx1519 & $12: 50$ & $14: 50$ & $t_{5}$ & 230 & $30 / 1800$ & $0 / 3600$ & $s_{1}$ & $s_{2}$ \\
\hline
\end{tabular}

Total delay minutes of flights: 550; canceled: 2 ; value of objective function: 226,587 .

TABle 3: Timetable after reassigning.

\begin{tabular}{|c|c|c|c|c|c|c|c|}
\hline$i$ & $f$ & $t_{f}^{d}$ & $t_{f}^{a}$ & $t_{i}$ & $E\left(t_{i}^{f}\right) /$ minute & $b_{f}^{d i}$ & $b_{f}^{a i}$ \\
\hline $737 / 3010$ & xx1337 & $9: 50$ & $14: 20$ & 11:00 & 70 & $s_{1}$ & $s_{3}$ \\
\hline $737 / 3010$ & xx1338 & $15: 30$ & 19:30 & $16: 10$ & 70 & $s_{3}$ & $s_{1}$ \\
\hline $737 / 3023$ & $\mathrm{xx} 1223$ & $12: 00$ & $16: 30$ & $12: 30$ & 30 & $s_{1}$ & $s_{3}$ \\
\hline $737 / 3023$ & xx1224 & $17: 00$ & $21: 30$ & $15: 30$ & 30 & $s_{3}$ & $s_{1}$ \\
\hline $310 / 8010$ & xx1518 & 8:00 & $12: 30$ & 8:00 & 0 & $s_{3}$ & $s_{1}$ \\
\hline $310 / 8010$ & xx1209 & $11: 00$ & $13: 30$ & 13:00 & 120 & $s_{1}$ & $s_{2}$ \\
\hline $310 / 3912$ & xx3108 & 8:00 & $10: 30$ & 8:00 & 0 & $s_{1}$ & $s_{2}$ \\
\hline $310 / 3912$ & xx3109 & 11:00 & $13: 30$ & 11:00 & 0 & $s_{2}$ & $s_{1}$ \\
\hline $310 / 3912$ & xx1519 & $15: 50$ & 14:50 & 14:00 & 70 & $s_{1}$ & $s_{2}$ \\
\hline $310 / 3912$ & xx1210 & $14: 00$ & $16: 30$ & $16: 30$ & 150 & $s_{2}$ & $s_{1}$ \\
\hline
\end{tabular}

Total delay minutes of flights: 540; , canceled: 0 ; value of objective function: 41,410.

Total cost of flights delay: 196,637 RMB.

algorithm to solve the problem. Compared with the traditional model, we introduce an uncertain variable into the model and construct it based on uncertain programming. We consider the delay minutes as uncertain variables with their uncertainty distributions given by experts. We construct a stepwise optimization method based on Hungarian algorithm to solve the model. From results of the numerical example, the total delay minutes of passengers are declined extensively, and we can get that the model and algorithm are feasible to deal with the issue of irregular flights.

A major contribution of this paper is that we provide a comprehensive framework for fleet reassignment during the period of irregular flights happen. Much work still needs to be done to improve on the current framework. Partially, we believe future research can be conducted on an integral framework of fleet reassignment and crew schedule recovery. Thus approaching the irregular flights problem is more systematical. Furthermore, considering actual situations, we can also construct an integral uncertain and stochastic model; thus, dealing with the issue of irregular flights is more comprehensive.

\section{Acknowledgments}

This work was supported by the Fundamental Research Funds for the Central Universities (Grant ZXH2011C008). The authors would like to thank the anonymous referees for useful suggestions and comments on the earlier draft of this paper.

\section{References}

[1] Z. Gao, D. Kong, and C. Gao, "Modeling and control of complex dynamic systems: applied mathematical aspects," Journal of Applied Mathematics, vol. 2012, Article ID 869792, 5 pages, 2012.

[2] A. Mathur and J. P. Clarke, How Healthy Is Your Operation, AGIFORS, 2005.

[3] D. Teodorović and S. Guberinić, "Optimal dispatching strategy on an airline network after a schedule perturbation," European Journal of Operational Research, vol. 15, no. 2, pp. 178-182, 1984.

[4] D. Teodorovic and G. Stojkovic, "Model to reduce airline schedule disturbances," Journal of Transportation Engineering, vol. 121, no. 4, pp. 324-331, 1995.

[5] A. I. Z. Jarrah, G. Yu, N. Krishnamurthy, and A. Rakshit, "Decision support framework for airline flight cancellations and delays," Transportation Science, vol. 27, no. 3, pp. 266-280, 1993.

[6] Y. Gang, “An optimization model for airlines' irregular operations control," in Proceedings of the International Symposium on Optimization Applications in Management and Engineering, 1995.

[7] S. Yan and D. Yang, "A decision support framework for handling schedule perturbation," Transportation Research B, vol. 30, no. 6, pp. 405-419, 1996. 
[8] M. F. Argüello, J. F. Bard, and G. Yu, "A GRASP for aircraft routing in response to groundings and delays," Journal of Combinatorial Optimization, vol. 1, no. 3, pp. 211-228, 1997.

[9] M. F. Argüello, J. F. Bard, and G. Yu, "Models and methods for managing airline irregular operations," International Series in Operations Research \& Management Science, vol. 9, pp. 1-45, 1998.

[10] J. F. Bard, G. Yu, and M. F. Argüello, "Optimizing aircraft routings in response to groundings and delays," IIE Transactions, vol. 33, no. 10, pp. 931-947, 2001.

[11] S. Bratu and C. Barnhart, "Flight operations recovery: new approaches considering passenger recovery," Journal of Scheduling, vol. 9, no. 3, pp. 279-298, 2006.

[12] P. Kouvelis and G. Yu, Robust Discrete Optimization and Its Applications, vol. 14, Kluwer Academic, Boston, Mass, USA, 1997.

[13] N. Matsveichuk, S. Yuri, and W. Frank, "Pertial job-Oorder for solving the two-machine flow-shop minimum-length problem with uncertain processing times," Information Control Problems in Manufacturing, vol. 13, no. 1, pp. 1517-1522, 2009.

[14] N. M. Matsveichuk, Y. N. Sotskov, and F. Werner, "The dominance digraph as a solution to the two-machine flow-shop problem with interval processing times," Optimization, vol. 60, no. 12, pp. 1493-1517, 2011.

[15] C. T. Ng, N. M. Matsveichuk, Y. N. Sotskov, and T. C. E. Cheng, "Two-machine flow-shop minimum-length scheduling with interval processing times," Asia-Pacific Journal of Operational Research, vol. 26, no. 6, pp. 715-734, 2009.

[16] B. Liu, Theory and Practice of Uncertain Programming, Springer, Berlin, Germany, 2nd edition, 2007.

[17] B. Liu, Uncertainty Theory, 4th edition, 2012.

[18] J. Peng and S. G. Li, "Spanning tree problem of uncertain network," http://orsc.edu.cn/online/110228.pdf.

[19] T. Andersson, "Solving the flight perturbation problem with meta heuristics," Journal of Heuristics, vol. 12, no. 1-2, pp. 37-53, 2006.

[20] Q. Gao, X. Tang, and J. Zhu, "Research on greedy simulated annealing algorithm for irregular flight schedule recovery model," in Advances in Grey Systems Research, pp. 503-513, Springer, Berlin, Germany, 2009.

[21] Z. Xiuli, Research on modeling and algorithm of airline irregular recovery [Ph.D. thesis], Nanjing University of Aeronautics and Astronautics, Nanjing, China, 2010.

[22] J. D. Petersen, G. Sölveling, J.-P. Clarke, E. L. Johnson, and S. Shebalov, "An optimization approach to airline integrated recovery," Transportation Science, vol. 46, no. 4, pp. 482-500, 2012. 


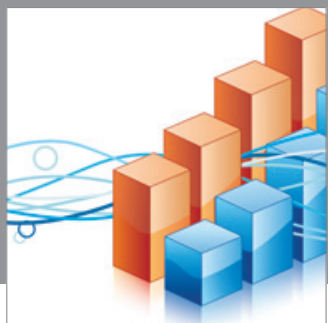

Advances in

Operations Research

mansans

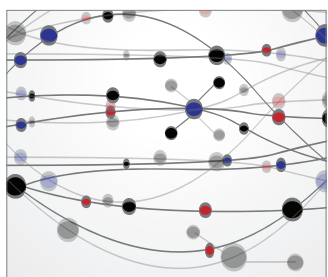

The Scientific World Journal
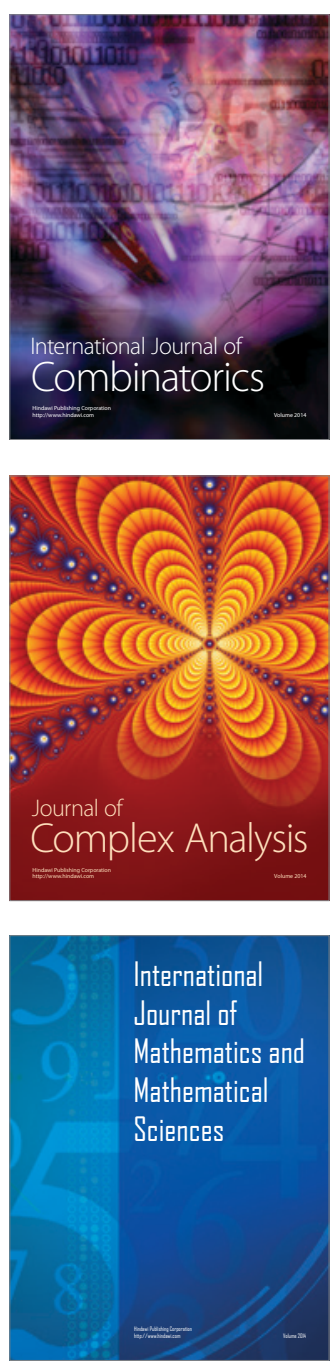
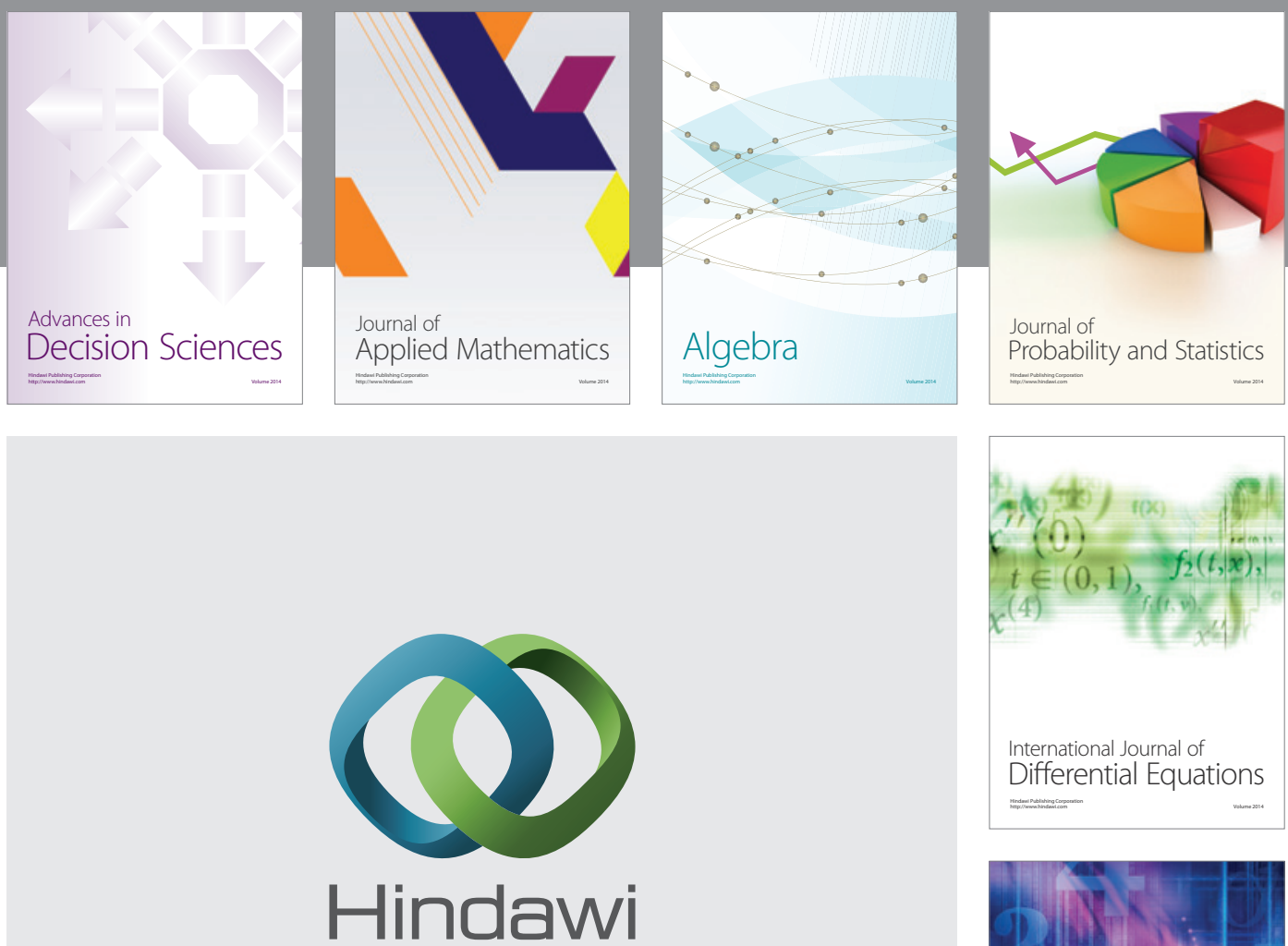

Submit your manuscripts at http://www.hindawi.com
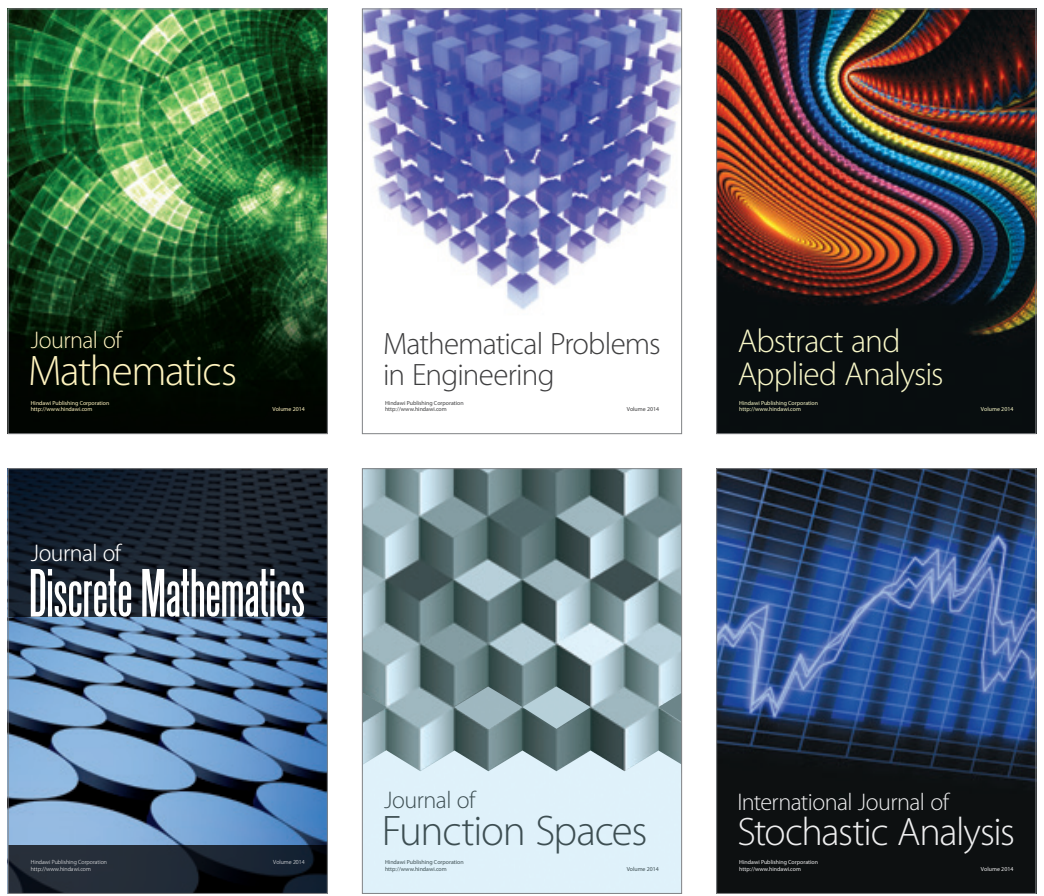

Journal of

Function Spaces

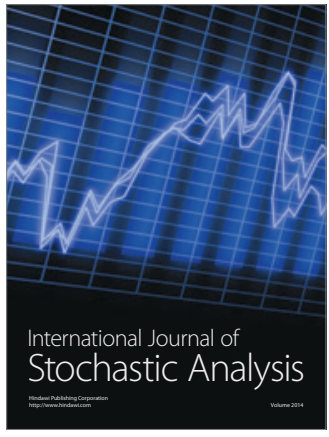

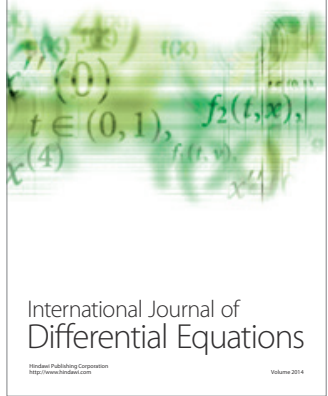
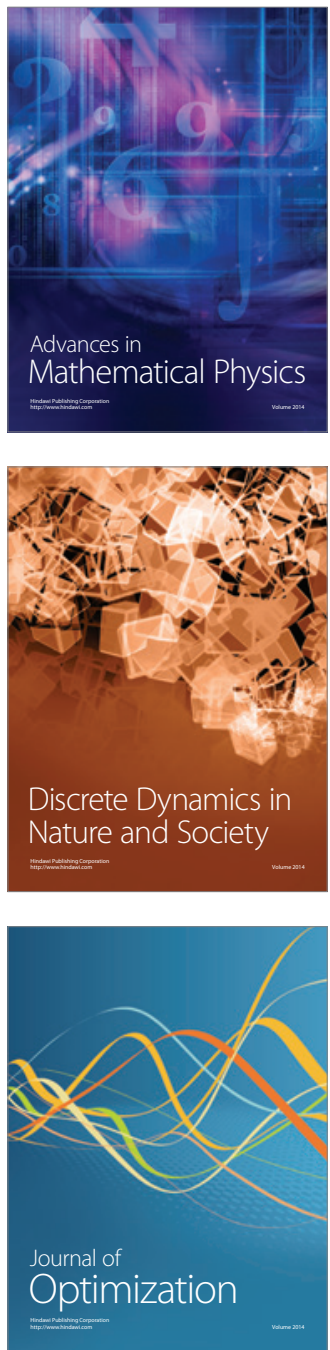